\title{
Cardiovascular magnetic resonance in hypertrophic cardiomyopathy and infiltrative cardiomyopathy
}

\begin{tabular}{|c|c|}
\hline \multicolumn{2}{|c|}{$\begin{array}{l}\text { Authors: } \\
\text { Rebecca Schofield }{ }^{1} \\
\text { Katia Manacho } \\
\text { Silvia Castelletti } \\
\text { James C. Moon } \\
\text { Ja,3 }\end{array}$} \\
\hline \multicolumn{2}{|c|}{$\begin{array}{l}\text { Affiliations: } \\
{ }^{1} \text { Barts Heart Centre, London, } \\
\text { United Kingdom }\end{array}$} \\
\hline \multicolumn{2}{|c|}{$\begin{array}{l}{ }^{2} \text { Istituto Auxologico Italiano } \\
\text { IRCCS 'San Carlo', Milan, Italy }\end{array}$} \\
\hline \multicolumn{2}{|c|}{$\begin{array}{l}{ }^{3} \text { Institute of Cardiovascular } \\
\text { Science, University College } \\
\text { London, United Kingdom }\end{array}$} \\
\hline \multicolumn{2}{|c|}{$\begin{array}{l}\text { Corresponding author: } \\
\text { James Moon, } \\
\text { j.moon@ucl.ac.uk }\end{array}$} \\
\hline \multicolumn{2}{|c|}{$\begin{array}{l}\text { Dates: } \\
\text { Received: } 02 \text { May } 2016 \\
\text { Accepted: } 09 \text { July } 2016 \\
\text { Published: } 11 \text { Nov. } 2016\end{array}$} \\
\hline \multicolumn{2}{|c|}{$\begin{array}{l}\text { How to cite this article: } \\
\text { Schofield R, Manacho K, } \\
\text { Castelletti S. \& Moon JC. } \\
\text { Cardiovascular magnetic } \\
\text { resonance in hypertrophic } \\
\text { cardiomyopathy and } \\
\text { infiltrative cardiomyopathy. S } \\
\text { Afr J Rad. 2016;20(2), a1020. } \\
\text { http://dx.doi.org/10.4102/ } \\
\text { sajr.v20i2.1020 }\end{array}$} \\
\hline \multicolumn{2}{|c|}{$\begin{array}{l}\text { Copyright: } \\
\text { (C) 2016. The Authors } \\
\text { Licensee: AOSIS. This } \\
\text { is licensed under the } \\
\text { Creative Commons } \\
\text { Attribution License. }\end{array}$} \\
\hline \multicolumn{2}{|l|}{ Read online: } \\
\hline 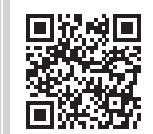 & $\begin{array}{l}\text { Scan this QR } \\
\text { code with your } \\
\text { smart phone or } \\
\text { mobile device } \\
\text { to read online. }\end{array}$ \\
\hline
\end{tabular}

Hypertrophic cardiomyopathy (HCM) is the most common inherited cardiac disease. Cardiac imaging plays a key role in the diagnosis and management, with cardiovascular magnetic resonance (CMR) an important modality. CMR provides a number of different techniques in one examination: structure and function, flow imaging and tissue characterisation particularly with the late gadolinium enhancement (LGE) technique. Other techniques include vasodilator perfusion, mapping (especially T1 mapping and extracellular volume quantification [ECV]) and diffusion-weighted imaging with its potential to detect disarray. Clinically, the uses of CMR are diverse. The imaging must be considered within the context of work-up, particularly the personal and family history, Electrocardiogram (ECG) and echocardiogram findings. Subtle markers of possible HCM can be identified in genotype positive left ventricular hypertrophy (LVH)-negative subjects. CMR has particular advantages for assessment of the left ventricle (LV) apex and is able to detect both missed LVH (apical and basal antero-septum), when the echocardiography is normal but the ECG abnormal. CMR is important in distinguishing HCM from both common phenocopies (hypertensive heart disease, athletic adaptation, ageing related changes) and rarer pheno and/or genocopies such as Fabry disease and amyloidosis. For these, in particular the LGE technique and T1 mapping are very useful with a low T1 in Fabry's, and high T1 and very high ECV in amyloidosis. Moreover, the tissue characterisation that is possible using CMR offers a potential role in patient risk stratification, as scar is a very strong predictor of future heart failure. Scar may also play a role in the prediction of sudden death. CMR is helpful in follow-up assessment, especially after septal alcohol ablation and myomectomy.

\section{Introduction}

Hypertrophic cardiomyopathy (HCM) is mainly an autosomal-dominant condition caused by mutations in sarcomeric proteins, with a prevalence of around 1 in 500 in the general population. First-degree relatives of known sarcomeric HCM patients have a one-in-two chance of developing HCM. Major advances in cardiac imaging and genetics over the last 50 years have improved our diagnostic ability in cases of HCM. The condition is linked to over 1500 genetic mutations, most commonly missense mutations in beta myosin heavy chain $(40 \%)$, myosin binding protein $\mathrm{C}$ sarcomere $(40 \%)$, and troponin $(11 \%)$ genes. ${ }^{1}$ Point mutations between affected individual families may be diverse. ${ }^{2}$ Genetic testing is helpful for family screening and also enables the exclusion of HCM mimics including storage disorders such as PRAGK2, Fabry's, Danon and LAMP2 which have very different prognosis and treatment options.

The clinical presentation and natural history of HCM may be equally as diverse, even between family members sharing the same mutation. Affected individuals may develop symptoms owing to left ventricular outflow tract obstruction (LVOTO) such as reduced exercise tolerance, dyspnoea, chest pain, pre-syncope and syncope on exertion. They may develop a heart failure syndrome owing to diastolic or systolic 'burnt out' dysfunction. Most concerning, they may have atrial arrhythmias (leading to stroke) or ventricular arrhythmias leading to syncope or sudden cardiac death. The ages of presentation vary widely. Some gene-positive family members may not express left ventricular hypertrophy ( $\mathrm{LVH}$ ) owing to incomplete penetrance, in part because of environmental factors and the presence or absence of other, as yet poorly defined, modifier genes.

The diagnosis of HCM may be acquired genetically (sarcomeric protein mutations), histologically (fibrosis, small vessel disease, disarray, no infiltration) or by cardiac morphology (unexplained hypertrophy). The clinical diagnosis is largely based on family history, ECG and cardiac imaging. Imaging has other roles beyond diagnosis, including risk stratification, defining features (i.e. obstruction) and subtype, detecting early disease and also in planning intervention. Echocardiography remains the first-line modality but CMR is becoming an essential next-line cardiac imaging test. 
In the present review, we focus on the role of CMR in the diagnosis and management of hypertrophic cardiomyopathy, heart muscle diseases with hypertrophy - HCM, and its gene and/or phenocopies.

\section{Utility of cardiovascular magnetic resonance}

CMR offers good spatial resolution and contrast between the myocardium and blood pool to accurately assess left ventricle (LV) wall thickness and mass. Short-axis slices from base to apex enable this assessment and also help to identify the subtype of HCM. Flow mapping can demonstrate LVOTO. Vasodilator (typically adenosine) stress can assess microvascular disease and coronary perfusion defects. Tissue characterisation by late gadolinium enhancement (LGE) imaging and parametric mapping can help to quantify the degree of replacement and interstitial fibrosis and differentiate HCM from other diseases causing $\mathrm{LVH}$ (i.e. infiltrative cardiomyopathies) and aid in risk stratification.

A standard CMR protocol for HCM involves a 10-minute assessment of anatomy and cardiac structure and function, followed by gadolinium-based contrast administration and scar imaging. Additional scanning may be added, such as thin $(5 \mathrm{~mm})$ slices through the apex or Left Ventricular Outflow Tract (LVOT), and additional sequences including parametric mapping (T1, T2 and ECV) can aid differentiation from cardiac amyloid, Fabry disease with cardiac involvement, diffuse myocardial inflammation and diffuse fibrosis. Flow mapping of the LVOT and cardiac valves can be used to assess obstruction and concomitant valve disease. The future may bring diffusion imaging (for disarray), spectroscopy and other metabolic imaging.

\section{Structure and function Volumes and ejection fraction}

The improved spatial resolution and the contrast between dark myocardium and bright blood pool on the steady-state free precession (SSFP) ciné enables accurate tracing of the epi- and endocardial borders. There are several areas of the heart that are better defined by CMR including the apex (Figure 1), ${ }^{3}$ early basal antero-septal hypertrophy (Figure 2$)^{4}$ and right ventricular hypertrophy (RVH). Wall thickness is well measured, but caution is needed as this is not well standardised by either echo or CMR, and typical risk algorithms use echocardiographic measurement. Similarly, normal values of volume and ejection fraction in CMR differ slightly from those calculated by echocardiography.

\section{Left ventricle crypts and subtle abnormalities}

The use of CMR extends the spectrum of detected cardiac changes in HCM. In patients with extensive T-wave inversion but non-diagnostic echo, relative apical hypertrophy may be found that does not meet current diagnostic criteria for HCM and can be demonstrated by CMR. Flett et al. ${ }^{3}$ described the lack of tapering of the LV wall thickness towards the apex in 22 patients with ECG abnormalities. An apical to basal wall thickness ratio $(\mathrm{ABR})>1$ is used as a criterion for diagnosis of relative apical hypertrophy (Figure 3). Presence of apical scar, left atrial dilatation and atrial fibrillation was also more common in this group than the general population, and apical micro-aneurysms (Figure 4 ) also occurred. This appears to be a variant of HCM, although there may be an environmental
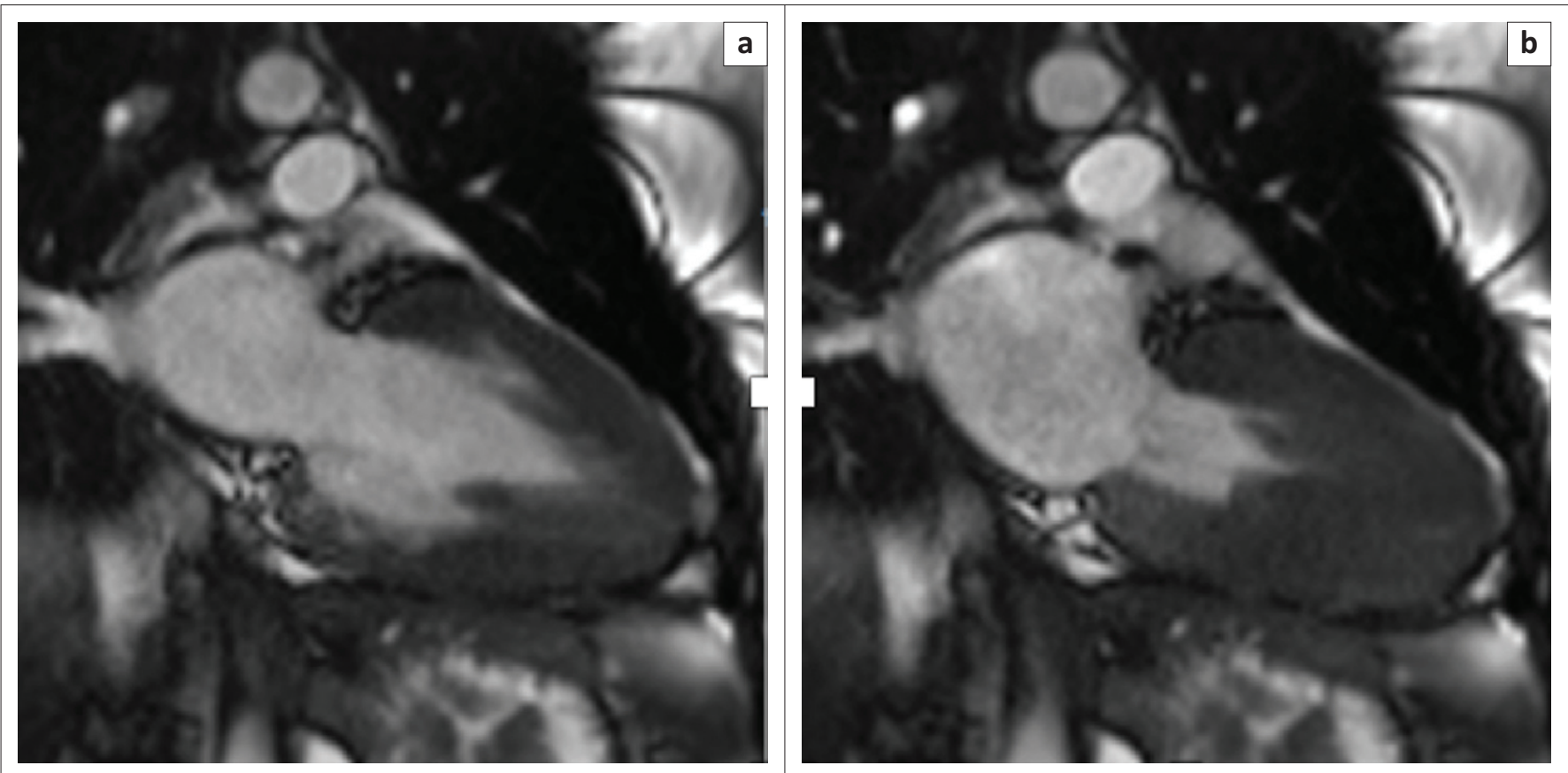

Source: Rebecca Schofield

FIGURE 1: Relative apical hypertrophy, seen in two-chamber view in diastole (a) and systole (b). Note also the basal inferior crypts. 
contribution as the pattern is found sometimes in athletes raising the suspicion that there are HCM genetic backgrounds where a 'second hit' is required for phenotypic expression.

Before hypertrophy occurs, the heart has a number of characteristic abnormalities if a disease-forming mutation is being carried. These include the presence of myocardial crypts (Figure 5). ${ }^{4}$ These appear to occur in the majority of genotype positive-phenotype negative HCM patients ${ }^{5}$ and may be multiple. They also occur in healthy volunteers (prevalence 3.6\% - 6\%) and hypertensive patients (prevalence $11 \%$ - 27\%). ${ }^{6,7}$ The prognostic importance of crypts is currently unclear. The number of crypts seems to reflect the degree of abnormality, with a maximum of one crypt seen in healthy volunteers and more than one (2-3) often seen in genetic carriage. ${ }^{8}$ A recent study of developing HCM hearts in mice and humans suggests that crypts are a normal part of embryological development but disappear before birth, except in HCM, where they persist and are more abundant. ${ }^{9}$

Increased length of the mitral valve leaflets has been identified in HCM and genotype positive-phenotype negative HCM independently of other variables, and this
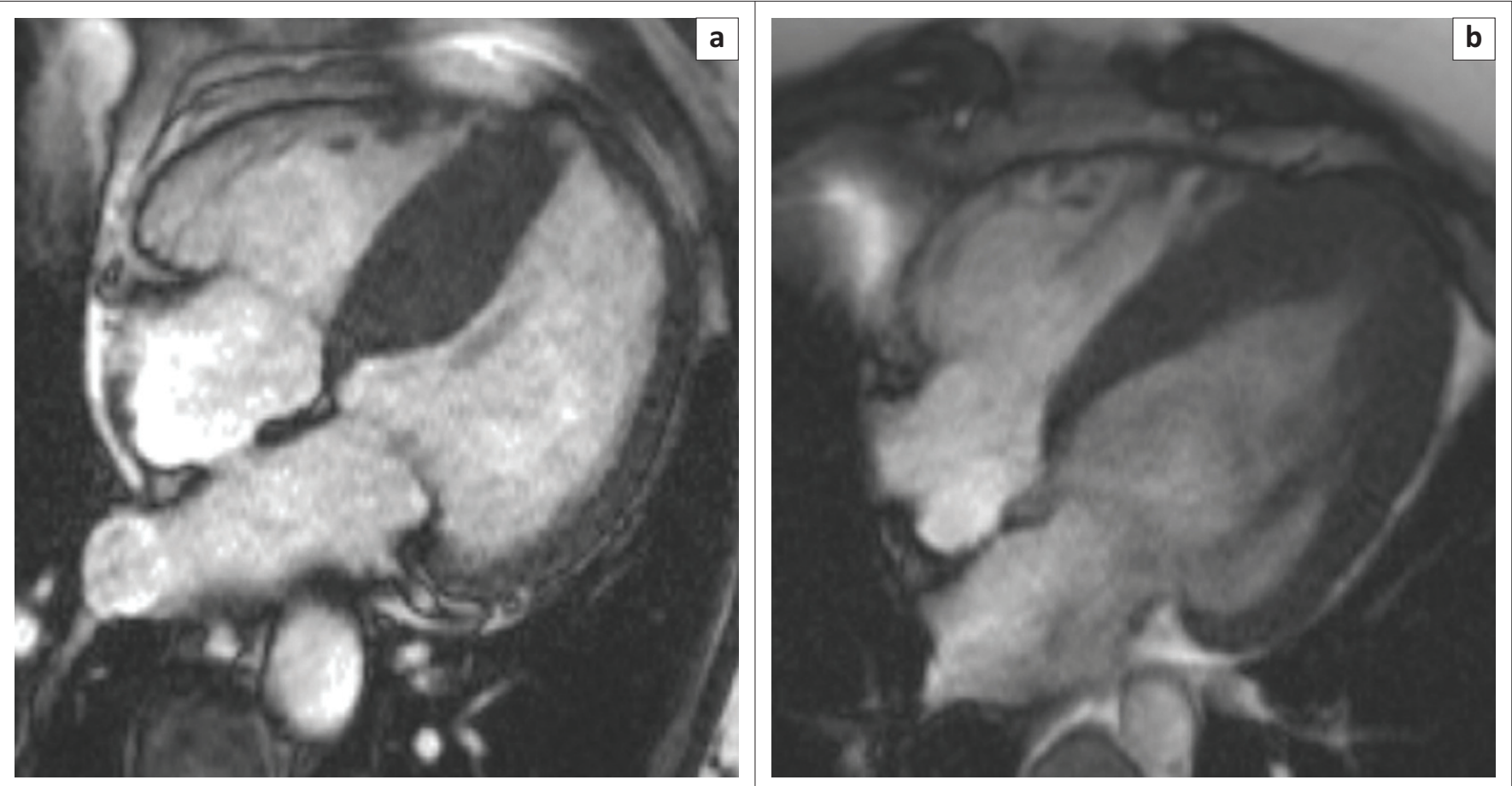

Source: Silvia Castelletti

FIGURE 2: Asymmetrical septal hypertrophy seen on a four-chamber ciné. Shown are the end diastolic (just after mitral valve [MV] closure) frame (a), and the end systolic frame (b).
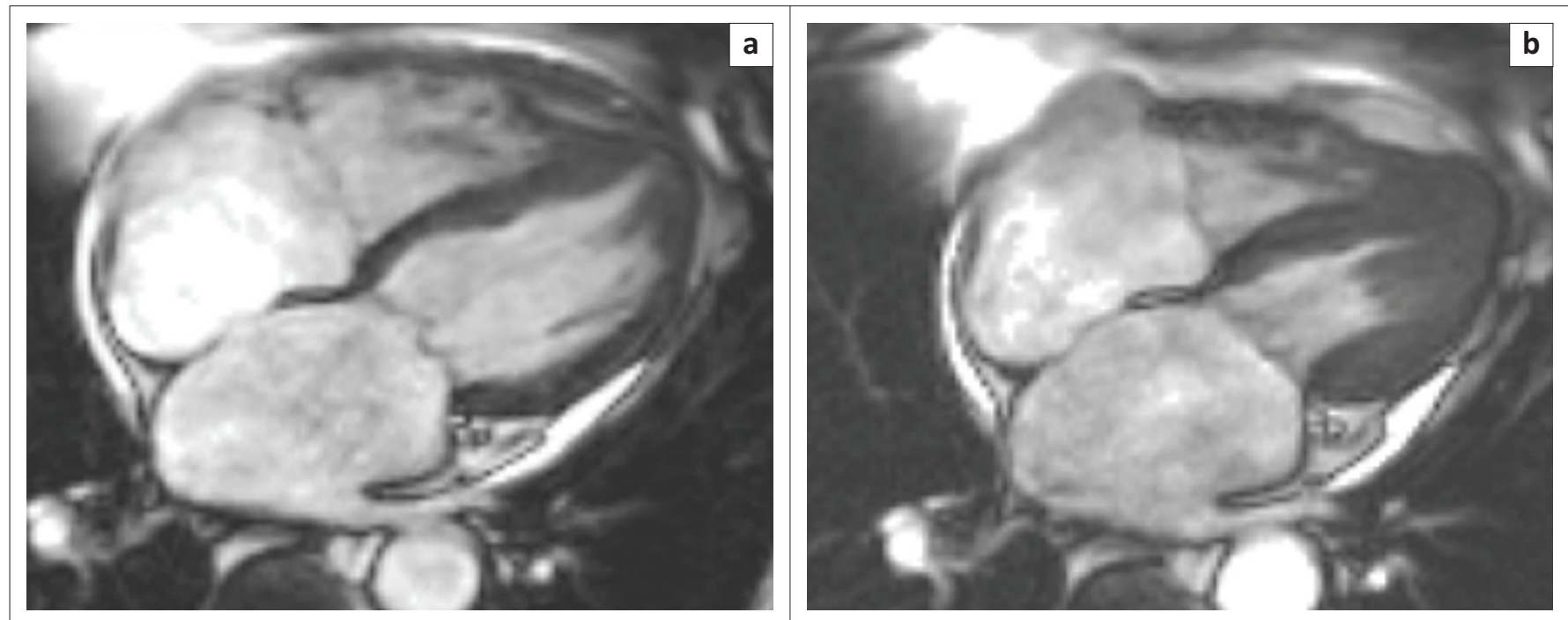

Source: Silvia Castelletti

FIGURE 3: Relative apical hypertrophy. Here, the normal tapering of wall thickness to the apex is absent and the maximum thickness is greater than that at the base. Also note the biatrial dilatation. Shown are the end diastolic frame (a), and the end systolic frame (b). 


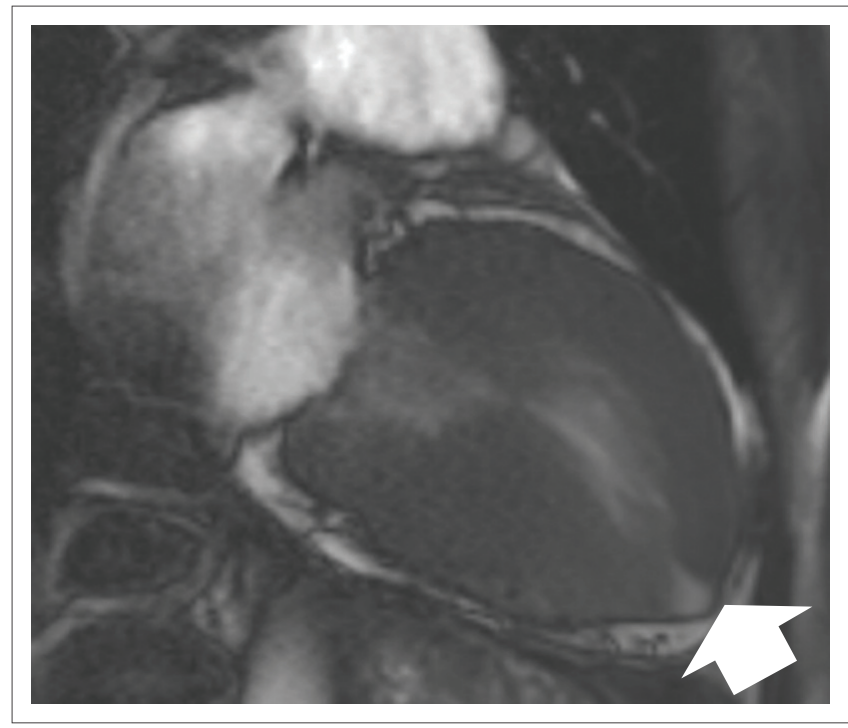

Source: Silvia Castelletti

FIGURE 4: An apical micro-aneurysm. These are hard to see by echocardiography in many cases.

additional morphological abnormality is felt to contribute to LVOTO. A ratio of anterior mitral leaflet (AML) length to LVOT diameter of $>2$ was strongly associated with the presence of sub-aortic obstruction. ${ }^{10}$ Other imaging biomarkers of HCM include increased septal curvature, increased cardiac function and more non-compacted trabeculae, abnormal papillary muscles, increased ECV and increased biomarkers of fibrosis. Several of these can be combined into a score that can have $80 \%$ accuracy for the presence of a disease (if the pre-test probability is $50 \%),{ }^{8,11}$

\section{Flow and perfusion}

Echocardiography is better for peak velocities and short time interval events. CMR, however, adds value in complex obstruction (e.g. multi-level) and membranes, which may be missed on echo. Similarly, right ventricular outflow tract (RVOT) obstruction is easier to detect using CMR than echo. For recurrent obstruction following gradient reduction therapy (by alcohol ablation or myomectomy), CMR is essential as the precise mechanism of recurrence may be hard to define by echocardiography. Perfusion CMR is interesting in HCM, as perfusion defects are often extensive and most often mid-myocardial and circumferential, suggesting they are the result of small vessel disease. These perfusion defects do occur outside areas of LGE, but their significance is not yet well understood. ${ }^{12}$

\section{Planning intervention}

When gradient reduction therapy is planned (alcohol ablation or myomectomy), CMR is helpful. It can define the systolic anterior motion (SAM), septal contact point and the wall thickness at the site and in adjacent areas ensuring particularly that there are no thin areas that are at risk of post-procedure ventricular septal defects (VSDs).

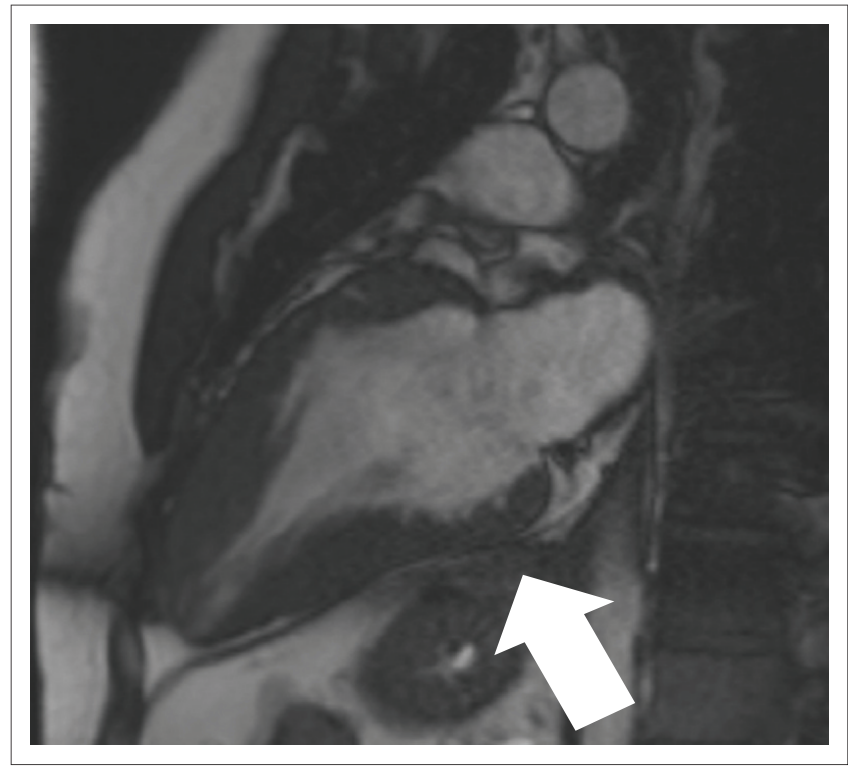

Source: James C. Moon

FIGURE 5: Basal inferior crypts; these are best seen in diastole. More than two is associated with cardiomyopathy.

CMR acts as a second scrutiny to ensure there is no membrane or aortic stenosis; it further helps with mitral valve assessment to determine whether mitral valve replacement or intervention (repair, Alfieri) might be needed.

Post alcohol septal ablation (ASA), CMR can assess procedural success by identifying and quantifying the area of septal infarction and the resultant effect on mitral valve function and LVOT obstruction (Figure 6). Post-procedural inter-ventricular thinning or procedural VSDs can be identified. Possibly owing to the relatively lower pressure in the right ventricle (RV) branches of the septal perforators, it is not uncommon to find that the RV portion of the intermyocardial septum has in fact infarcted preferentially to the LV portion (and asymmetric septal hypertrophy [ASH] gives right bundle branch block [RBBB], myomectomy typically left bundle branch block [LBBB]).

\section{Tissue characterisation}

\section{Late gadolinium enhancement patterns}

Gadolinium occupies the interstitium and cannot enter an intact myocyte. Fibrosis is a hallmark of HCM. ${ }^{13}$ Hypothesised aetiologies include repeated ischaemic insults owing to microvascular disease and oxygen supply and demand mismatch; alternatively, the genetic mutation may predispose to the abnormal accumulation of collagen within the myocardium and plexiform diffuse fibrosis owing to an increased number of collagen crosslinking fibres at the LV/RV hinge points. Typical patterns of LGE include 'non-ischaemic' (mid-myocardial) fibrosis of the maximally hypertrophied segments which may include the $\mathrm{LV} / \mathrm{RV}$ insertion points or commonly the septum and anteroseptal walls (Figure 7). There may be a combination of diffuse interstitial fibrosis and focal replacement fibrosis. 


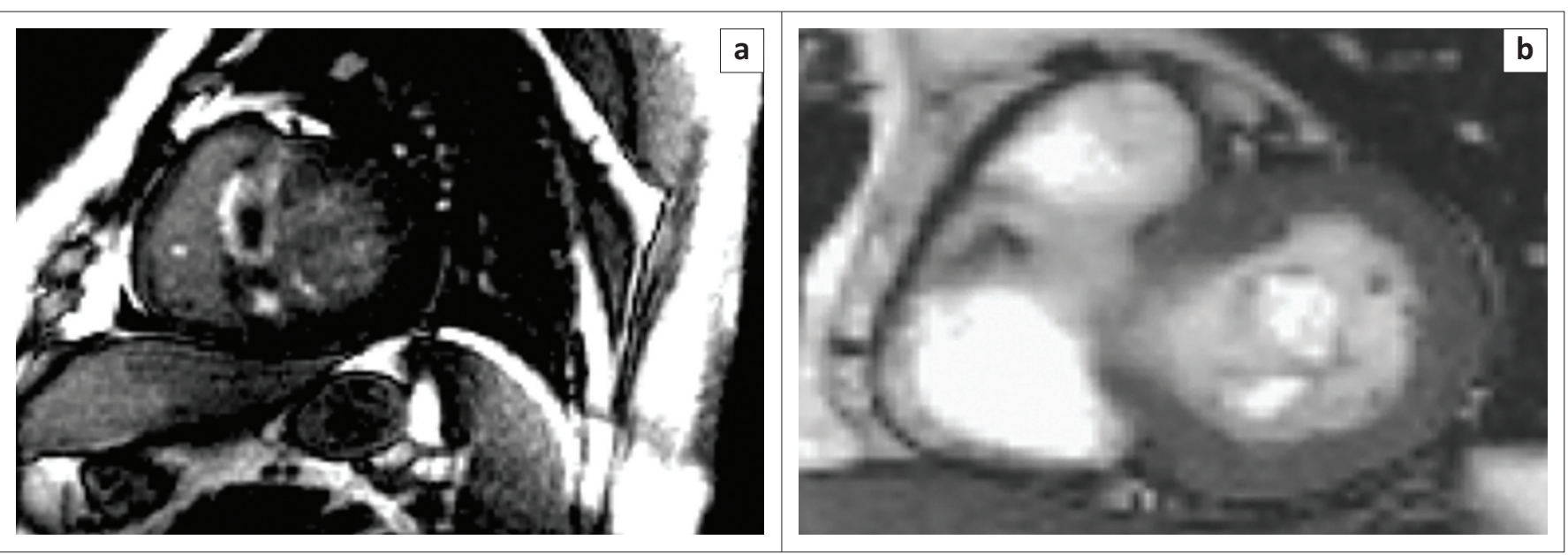

Source: James Moon

FIGURE 6: (a) An alcohol ablation that is acute (microvascular obstruction at the core with a limited scar in the moderator band which may also have been an 'off-target' consequence. (b) A myectomy (ciné still in diastole) with Alfieri stitch.
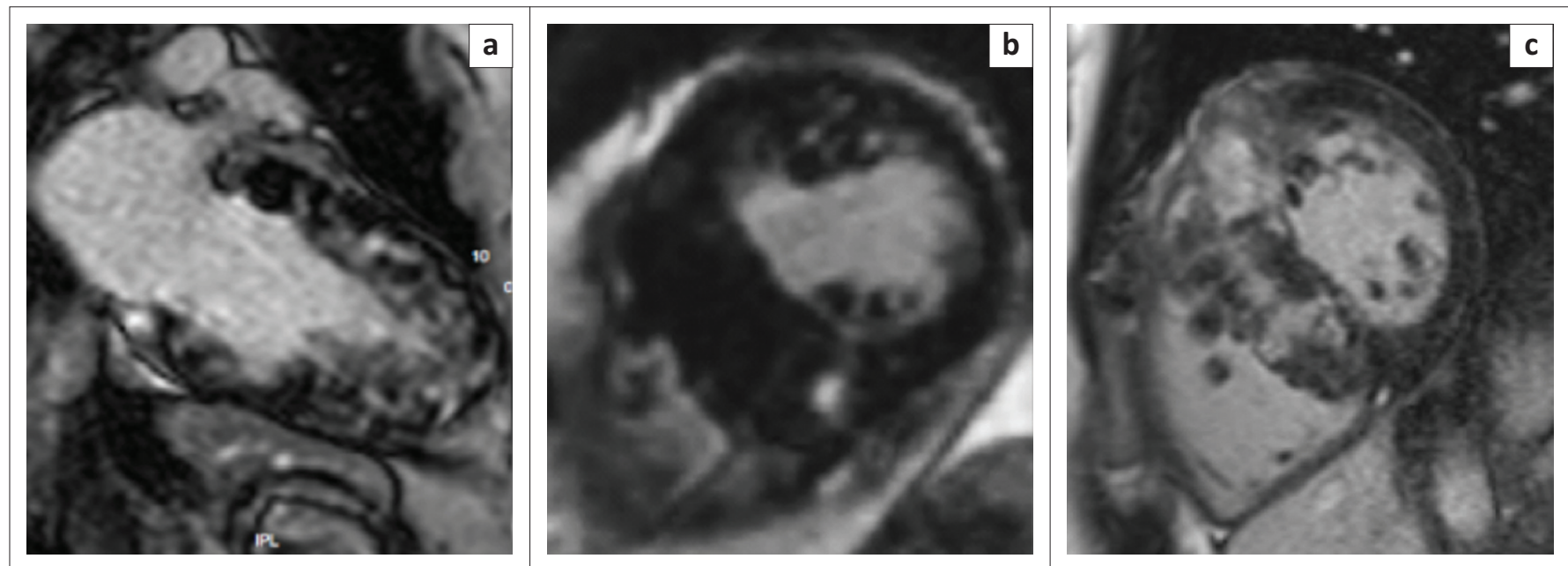

Source: Silvia Castelletti

FIGURE 7: Patterns of LGE. (a) Two-chamber view in apical HCM-note heterogeneous LGE in mid to apical LV. (b) Mid short-axis (SAX) slice in septal HCM-focal inferior LV/ RV insertion point LGE. (c) Basal SAX in septal HCM-patchy septal LGE.

The replacement fibrosis may, in progressive disease cases, involve most of the myocardial walls.

\section{Parametric mapping}

Parametric mapping is another technique that helps to characterise tissue. The myocardial native T1 value increases in the presence of water and protein, and is reduced by the presence of fat and iron. ECV maps can be created from $\mathrm{T} 1$ values pre and post gadolinium contrast (Figure 8). The strength of LGE imaging is in demonstrating the difference between normal and abnormal myocardium, the best example being non-infarcted from infarcted myocardium where there is stark contrast between healthy (black myocardium) and dead tissue (white myocardium). In conditions of diffuse myocardial abnormality, choosing a correct inversion time (TI) to null the healthy myocardium can result in both fibrotic and normal myocardium looking similar and, consequently, abnormality being missed. Techniques for quantifying the amount of LGE can show wide variations in the figures generated when used to assess more subtle replacement and interstitial fibrosis.

\section{Risk stratification}

HCM sudden death is relatively uncommon but feared - the lack of warning signs and the finality of the event make it of extreme concern. Typically, it occurs in the younger HCM population, being less likely over the age of 65 years. With the advent of implantable cardiac defibrillators (ICDs), sudden cardiac death (SCD) may be prevented; however, the devices themselves carry a risk (approximately $5 \%$ per year) related to infection, lead defects and inappropriate shocks. Appropriate patient selection is important. Secondary prevention is essential but, for others, risk stratification is needed. These models are created using large retrospective cohort studies and are evolving over time. The European Society of Cardiology (ESC) risk calculator aims to give an approximate 5 year risk of SCD. In patients with a 5 year risk of SCD $<4 \%$, an ICD is generally not indicated, in patients with a risk of 4 to less than $6 \%$, an ICD may be considered and in patients with a 5 year risk $\geq 6 \%$, an ICD should be considered. The algorithm includes several continuous variables (age, maximal wall thickness by echo, left atrial size, maximum LVOT gradient), 

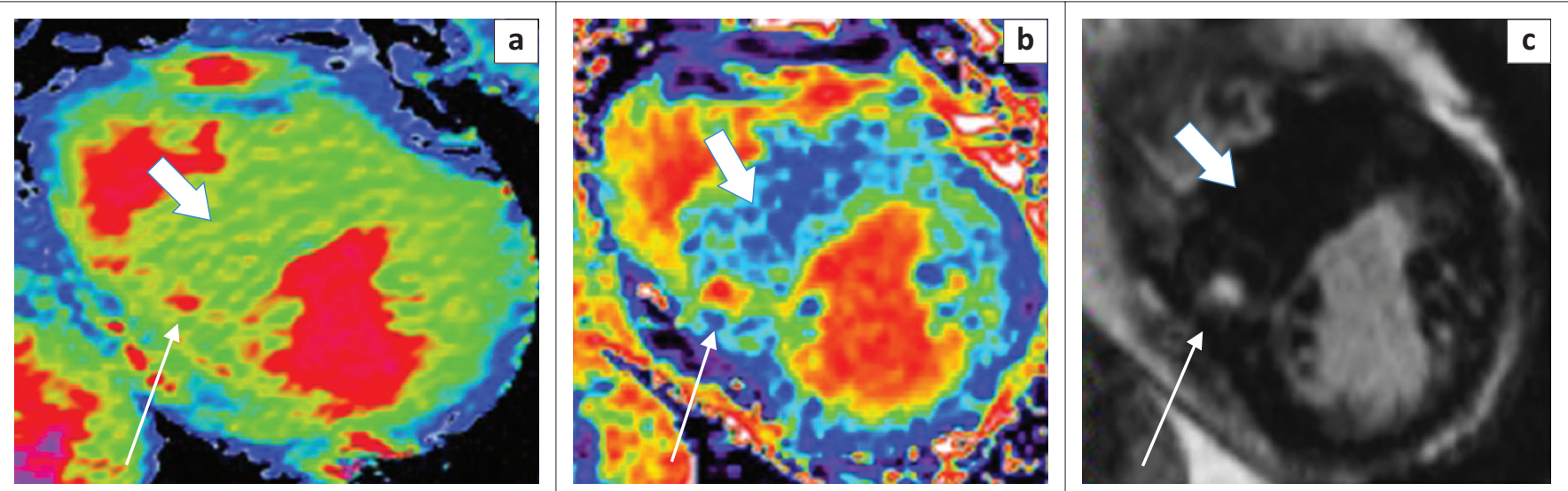

Source: Katia Manacho

FIGURE 8: Native T1 map (a), ECV map (b) and LGE in mid SAX slice (c) in HCM. Note the inferior LV/RV insertion point fibrosis seen as high T1 and high ECV volume and LGE respectively. The LGE image sees the inferior RV insertion point LGE (long arrow) but misses the wider patchy fibrosis (short arrows) in the maximally hypertrophied septal segments which is easily appreciated in the native T1 and ECV maps.

and three dichotomous ones (family history of SCD, previous non-sustained VT (ventricular tachycardia) and unexplained syncope). The AHA (American Heart Association) guidelines acknowledge the potential use of LGE by CMR as a risk modifier, with Class Ilb evidence in risk stratification.

CMR to assess myocardial scar and fibrosis is an attractive additional variable as fibrosis appears to be linked to outcome. ${ }^{14,15,16}$

Fibrosis and disruption of the myocardial architecture has been suggested as the substrate for SCD, leading to research quantifying the degree of fibrosis and the relationship to SCD and VT. The LGE analysis technique assesses macroscopic fibrosis, and all are subject to the difficulties in TI (inversion time) selection, windowing and detection of diffuse fibrosis discussed earlier.

What is clear, however, is that scar is common in HCM occurring in $60 \%-70 \%$ of cases - so scar alone is not sufficient for adverse outcomes. There have been several studies with numbers into the low 1000s. The link of scar to sudden death appears present although, in some studies, this was present as a univariate but not multivariate risk factor; in one study by O'Hanlon et al., ${ }^{15}$ the presence of non-sustained VT was the stronger factor, whilst in another by Chan et al., ${ }^{16}$ scar over 15\% predicted events, although events were driven by appropriate ICD discharges (not the same as sudden death). ${ }^{16}$ What does appear clear, however, is that extensive scar predicts heart failure; the problem is that the evidence for interventions (drug therapy - conventional or otherwise) is not strong and more research is needed. Another issue is LGE analysis. Although reasonable agreement can be reached, ${ }^{17}$ the choice of method can alter the apparent scar burden by a factor of two in HCM. ${ }^{18}$ This reflects that scar in HCM is in a continuum between normal interstitium and complete replacement fibrosis, yet the LGE technique dichotomises with a threshold method that therefore becomes crucial. Analysis on commercially available software (rather than corelabs) would be more clinically relevant, and newer LGE sequences (phase-sensitive inversion recovery [PSIR], MOCO and implantable device corrected) are more robust than previous LGE sequences.

All studies thus far have quantified LGE on a single-entry CMR only. Rate of progression of LGE may be of importance, as may myocardial ischaemia. More data are needed. Currently, CMR is typically used as a red flag or arbitrator for borderline cases rather than a core part of stratification.

\section{Hypertrophic cardiomyopathy phenocopies and infiltrative cardiomyopathies}

Sarcomeric HCM has a wide differential. Approaches to heart muscle disease with hypertrophy are familiar from European and US guidelines (taking phenotype or genetic approaches respectively). Both approaches are useful, but 'grey areas' remain. CMR tissue characterisation permits a new way of considering $\mathrm{LVH}$ by dividing it into two compartments: the interstitium and cell volume. Usually, the volume of interstitium and cells increase together (afterload hypertension and aortic stenosis), but in some diseases (amyloid), the interstitial volume predominates and in others (storage diseases - at least early, such as Fabry's), cell volume appears to predominate.

Differentiating causes of LVH by imaging is familiar from echocardiography, and these features are visible by CMR. Imaging, of course, is just part of investigation, and the history (including family history), ECG, exercise testing and other investigations are important. CMR adds some value with the added features for HCM (clefts) and may see early disease better than echo (focal hypertrophy). When differentiating hypertension or athlete's heart from HCM, mapping may help; early data suggest that athletic hypertrophy has a slightly lower than normal fibrosis percentage (low ECV, low T1) with cellular hypertrophy dominating. ${ }^{19}$ These tissue characterisation techniques, however, have a major advantage in infiltration. 


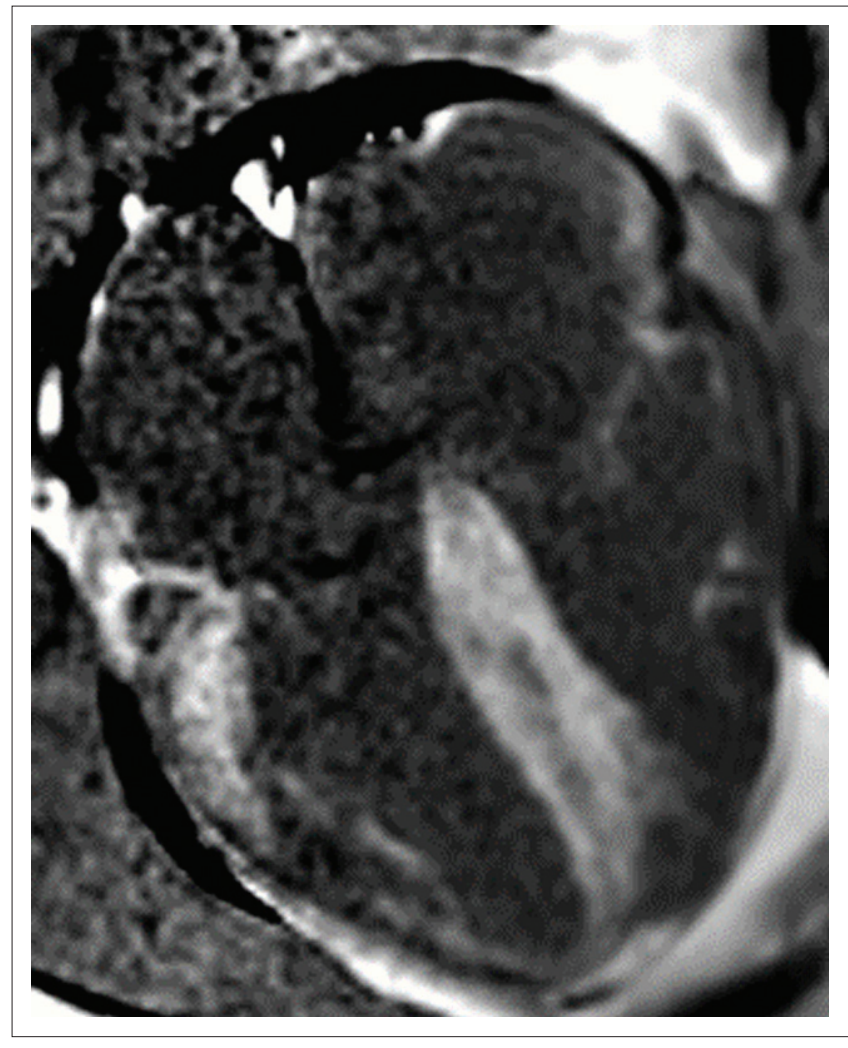

Source: James Moon

FIGURE 9: Four-chamber LGE sequence in cardiac amyloid. The gadolinium kinetics are abnormal. Blood pool nulls before myocardium (which appears bright).

\section{Infiltration: Cardiac amyloid and Fabry disease}

In cardiac amyloid, gadolinium kinetics are abnormal and the blood pool nulls before the myocardium (Figure 9). Choosing the appropriate TI value can be challenging and, if incorrect, can result in misleading LGE images. The PSIR technique solves this problem. ${ }^{20}$ LGE starts subendocardially, becoming transmural initially at the base of the LV before extending down to the apex. Transmural LGE confers a poor prognosis in both Amyloid light-chain (AL) and transthyretinrelated amyloidosis (ATTR). When there is subendocardial LGE, outcomes are intermediate and research is currently under way to determine the best treatments for this group. Cardiac amyloid can be detected without using gadolinium contrast if native $\mathrm{T} 1$ values are significantly elevated throughout the myocardium (typically six to eight standard deviations above normal). The native $\mathrm{T} 1$ can be used as an early marker disease, to differentiate subtypes (AL and ATTR) and as a prognostic tool. ${ }^{21}$

Most storage diseases (glycogen, lysomal) appear to cause LV scar, but the natural history of these diseases is not well known. In Fabry disease, an X-linked disorder of sphingolipid metabolism, there are more specific features: the basal inferolateral wall typically shows mid-wall LGE (Figure 10) and the native $\mathrm{T} 1$ values are lower than the normal range. Moreover, it has been shown that the T1 value can be an early marker of disease in Fabry's patients, even in the absence of hypertrophy. ${ }^{22}$ Recent work measuring T2 values in areas of LGE has demonstrated high T2 values that correlate with

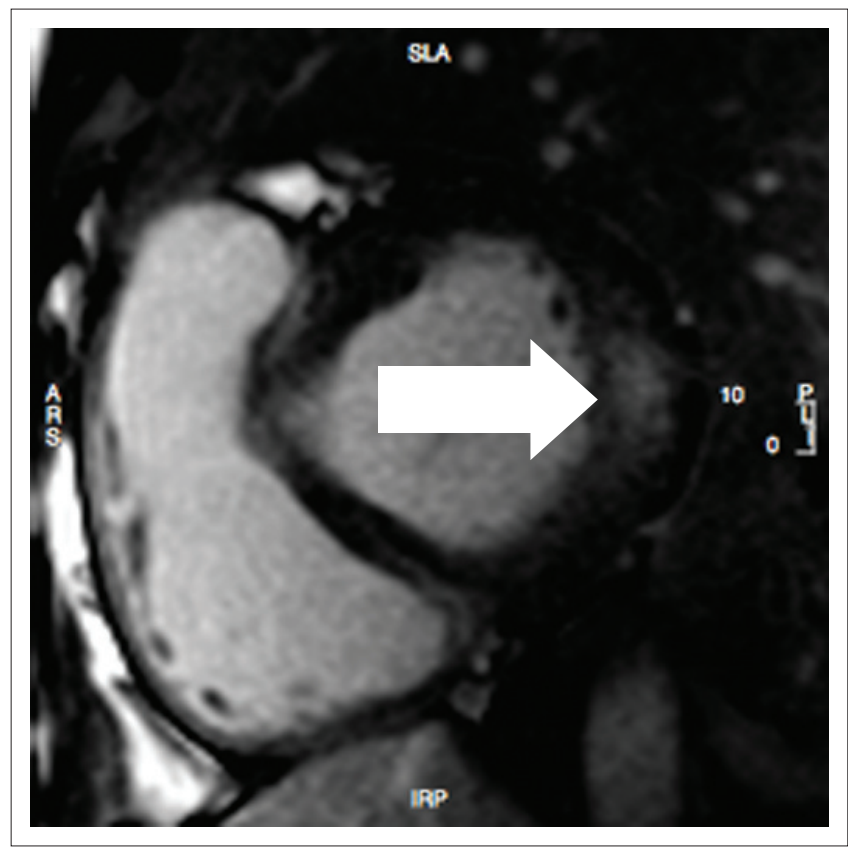

Source: Rebecca Schofield

FIGURE 10: Basal lateral mid-wall LGE in Fabry disease. The differential for this scar pattern includes previous myocarditis, which T1 mapping would confirm.

high-sensitivity troponin $\mathrm{T}$ (hs-TnT), suggesting a possible chronic inflammatory component.

\section{Other forms of infiltration: Sarcoidosis and iron}

Perhaps implied by the history, cardiac sarcoid can be detected by CMR. Acutely, there may be myocardial thickening and evidence of oedema on T2-weighted Short TI Inversion Recovery (STIR) imaging or T2 mapping. There may be patchy focal epicardial, transmural or mid-wall LGE and RV involvement. T2* imaging is exquisitely specific for myocardial iron deposition but the diagnostic cut-off may be set too high. Refinement of new diagnostic thresholds may be possible using concomitant native T1 values. Characteristically, there is no wall thickening and low T2* and $\mathrm{T} 1$ values. Fabry disease and iron are largely the main causes of low $\mathrm{T} 1$.

\section{Limitations of cardiovascular magnetic resonance}

Although newer scanners have a larger bore, there are still patients who cannot tolerate the confined space of the scanner. In addition, there are patients where image degradation is intolerable and results in non-diagnostic image quality owing to arrhythmias and difficulty with breath-holding.

Patients with cardiac devices, including pacemakers and ICDs, may have non-MR compatible devices or leads. In those who have compatible devices, the artefact from the device can obscure the images, rendering the images uninterpretable.

Newer sequences to address some of these issues are in progress. 


\section{Future developments}

Although CMR now offers significant utility, there are problems. CMR is expensive and access is limited. Choosing the right patients to scan for maximal cost-effectiveness is not easy. Protocols may need to become shorter, less involved for patients and more focused. Standardisation is needed for the latest techniques (such as T1 mapping), which may conflict with efforts to continue development. For example, T1 mapping sequences which do not require breath hold are being developed, and motion-corrected (MOCO) freebreathing LGE sequences are in clinical use in some centres that may enable the visualisation of more detail. Nevertheless, CMR roll-out is continuing and should continue to inform our understanding of disease, particularly in hypertrophy. Newer techniques are pending, such as diffusion tensor imaging (DTI) to visualise fibre architecture that may help our understanding of myocardial remodelling; and 4D flow is beginning to inform on cardiac haemodynamic and energetic efficiency during rest and exercise.

\section{Conclusion}

CMR is pushing the envelope in HCM both in terms of early and more accurate diagnosis and a better understanding of the conditions and their true pathogenesis. Use of tissue characterisation techniques, such as LGE and parametric mapping, provides detailed information about the myocytes and cellular matrix in vivo that is beginning to translate into better care and treatment choices, to the patient's benefit.

\section{Competing interests}

The authors declare that they have no financial or personal relationships which may have inappropriately influenced them in writing this article.

\section{Authors' contributions}

S.C. and K.M. contributed images. The text was written by R.S. and J.M., with contributions from S.C., and was reviewed by S.C.

\section{References}

1. Maron BJ, Maron MS, Semsarian C. Genetics of hypertrophic cardiomyopathy after 20 years: Clinical perspectives. J Am Coll Cardiol. 2012;60:705-715. http:// dx.doi.org/10.1016/j.jacc.2012.02.068

2. Seidman CE, Seidman JG. Identifying sarcomere gene mutations in hypertrophic cardiomyopathy: A personal history. Circ Res. 2011;108:743-750. http://dx.doi. org/10.1161/CIRCRESAHA.110.223834
3. Flett A, Maestrini V, Miliken D, et al. Diagnosis of apical hypertrophic cardiomyopathy: T-wave inversion and relative but not absolute apical left ventricular hypertrophy. Int J Cardiol. 2015;183:143-148. http://dx.doi.org/10. 1016/j.ijcard.2015.01.054

4. Maron MS, Rowin EJ, Lin D, et al. Prevalence and clinical profile of myocardial crypts in hypertrophic cardiomyopathy. Circ Cardiovasc Imaging. 2012;5:441-447. http://dx.doi.org/10.1161/CIRCIMAGING.112.972760

5. Germans T, Wilde AA, Dijkmans PA, et al. Structural abnormalities of the inferoseptal left ventricular wall detected by cardiac magnetic resonance imaging in carriers of hypertrophic cardiomyopathy mutations. J Am Coll Cardiol. 2006;48:2518-2523. http://dx.doi.org/10.1016/j.jacc.2006.08.036

6. Johansson B, Maceira AM, Babu-Narayan SV, Moon JC, Pennell DJ, Kilner PJ. Clefts can be seen in the basal inferior wall of the left ventricle and the interventricula septum in healthy volunteers as well as patients by cardiovascular magnetic resonance. J Am Coll Cardiol. 2007;50:1294-1295. http://dx.doi.org/10.1016/j. jacc.2007.06.026

7. Child N, Muhr T, Sammut E, et al. Prevalence of myocardial crypts in a large retrospective cohort study by cardiovascular magnetic resonance. J Cardiovasc Magn Reson. 2014;16:66. http://dx.doi.org/10.1186/s12968-014-0066-0

8. Captur G, Lopes L, Patel V, et al. Abnormal cardiac formation in hypertrophic cardiomyopathy: Fractal analysis of trabeculae and preclinical gene expression Circ Cardiovasc Genet. 2014;7(3):241-248. http://dx.doi.org/10.1161/CIRCGEN ETICS.113.000362

9. Captur G, Ho CY, Schlossarek S, et al. The embryological basis of subclinical hypertrophic cardiomyopathy. Sci Rep. 2016;6:27714. http://dx.doi.org/10.1038/ srep27714

10. Maron M, Olivotto I, Harrigan C, et al. Mitral valve abnormalities identified by cardiovascular magnetic resonance represent a primary phenotype expression of hypertrophic cardiomyopathy. Circualtion. 2011;124:40-47. http://dx.doi.org/ 10.1161/CIRCULATIONAHA.110.985812

11. Captur G, Lopes LR, Mohun TJ, et al. Prediction of sarcomere mutations in subclinical hypertrophic cardiomyopathy. Circ Cardiovasc Imaging. 2014;7(6):863871. http://dx.doi.org/10.1161/CIRCIMAGING.114.002411

12. Ismail TF, Hsu LY, Greve AM, et al. Coronary microvascular ischemia in hypertrophic cardiomyopathy - A pixel-wise quantitative cardiovascular magnetic resonance perfusion study. J Cardiovasc Magn Reson. 2014;16:49. http://dx.doi.org/10.1186/ s12968-014-0049-1

13. Davies MJ, McKenna WJ. Hypertrophic cardiomyopathy - Pathology and pathogenesis. Histopathol. 1995;26:493-500. http://dx.doi.org/10.1111/j. 1365-2559.1995.tb00267.x

14. Maron MS, Appelbaum E, Harrigan CJ, et al. Clinical profile and significance of delayed enhancement in hypertrophic cardiomyopathy. Circ Heart Fail. 2008;1:184-191. http://dx.doi.org/10.1161/CIRCHEARTFAILURE.108.768119

15. O'Hanlon R, Grasso A, Roughton $M$, et al. Prognostic significance of myocardial fibrosis in hypertrophic cardiomyopathy. J Am Coll Cardiol. 2010;56:867-874. http://dx.doi.org/10.1016/j.jacc.2010.05.010

16. Chan RH, Maron BJ, Olivotto I, et al. Prognostic value of quantitative contrastenhanced cardiovascular magnetic resonance for the evaluation of sudden death risk in patients with hypertrophic cardiomyopathy. Circulation. 2014;130:484-495. $\mathrm{http} / / / \mathrm{dx}$.doi.org/10.1161/CIRCULATIONAHA.113.007094

17. Ismail T, Jabbour A, Gulati A, et al. Role of late gadolinium enhancement cardiovascular magnetic resonance in the risk stratification of hypertrophic cardiomyopathy. Heart. 2014;100:1-8. http://dx.doi.org/10.1136/heartjnl-2013-305471

18. Flett AS, Hasleton J, Cook C, et al. Evaluation of techniques for the quantification of myocardial scar of differing etiology using cardiac magnetic resonance. JACC Cardiovasc Imaging. 2011;4(2):150-156. http://dx.doi.org/10.1016/j.jcmg.2010. Cardiov 11.015

19. McDiarmid AK, Swoboda PP, Erhayiem B, et al. Athletic cardiac adaptation in males is a consequence of elevated myocyte mass. Circ Cardiovasc Imaging. 2016;9(4):pii:e003579. http://dx.doi.org/10.1161/CIRCIMAGING.115.003579

20. Fontana $M$, Pica $S$, Reant $P$, et al. Prognostic value of late gadolinium enhancement cardiovascular magnetic resonance in cardiac amyloidosis. Circulation. 2015; 132(16):1570-1579. http://dx.doi.org/10.1161/CIRCULATIONAHA.115.016567

21. Banypersad SM, Fontana M, Maestrini V. T1-mapping and survival in systemic light-chain amyloidosis. Eur Heart J. 2015;36:244-251. http://dx.doi.org/10.1093/ eurheartj/ehu444

22. Sado DM, White SK, Piechnik SK. Identification and assessment of Anderson-Fabry disease by cardiovascular magnetic resonance non-contrast myocardial T1 mapping. Circ Cardiovasc Imaging. 2013;6(3):392-398. http://dx.doi.org/10.1161/ CIRCIMAGING.112.000070 